\title{
Bilinearization and Casorati determinant solution to the non-autonomous discrete $\mathrm{KdV}$ equation
}

\author{
Kenji KaJiwara \\ Faculty of Mathematics, Kyushu University, 6-10-1 Hakozaki, Fukuoka 812-8581, Japan \\ YASUHIRO OHTA \\ Department of Mathematics, Kobe University, Rokko, Kobe 657-8501, Japan
}

\begin{abstract}
Casorati determinant solution to the non-autonomous discrete $\mathrm{KdV}$ equation is constructed by using the bilinear formalism. We present three different bilinear formulations which have different origins.
\end{abstract}

\section{Introduction}

In this article, we consider the following partial difference equation

$$
\left(\frac{1}{a_{m}}+\frac{1}{b_{n+1}}\right) v_{n+1}^{m}-\left(\frac{1}{a_{m+1}}+\frac{1}{b_{n}}\right) v_{n}^{m+1}=\left(\frac{1}{a_{m}}-\frac{1}{b_{n}}\right) \frac{1}{v_{n}^{m}}-\left(\frac{1}{a_{m+1}}-\frac{1}{b_{n+1}}\right) \frac{1}{v_{n+1}^{m+1}},
$$

where $m, n$ are the discrete independent variables, $v_{n}^{m}$ is the dependent variable on the lattice site $(m, n)$, and $a_{m}, b_{n}$ are arbitrary functions of $m$ and $n$, respectively. Recently eq. (1) has been derived by Matsuura[1] as the equation of motion of discrete curves on the centro-affine plane. In this context, $v_{n}^{m}$ is related to the curvature and it is essential that $a_{m}$ and $b_{n}$ depend on $m$ and $n$, respectively. For analyzing the motion of discrete curve, constructing solutions of eq.(1) explicitly is an interesting subject. We call eq.(1) the non-autonomous discrete $\mathrm{KdV}$ equation, for if $a_{m}$ and $b_{n}$ are constants, e.g. $a_{m}=a, b_{n}=b$, eq. (1) reduces to the discrete KdV equation[2, 3]

$$
\left(\frac{1}{a}+\frac{1}{b}\right) v_{n+1}^{m}-\left(\frac{1}{a}+\frac{1}{b}\right) v_{n}^{m+1}=\left(\frac{1}{a}-\frac{1}{b}\right) \frac{1}{v_{n}^{m}}-\left(\frac{1}{a}-\frac{1}{b}\right) \frac{1}{v_{n+1}^{m+1}}
$$

or

$$
v_{n+1}^{m}-v_{n}^{m+1}=\frac{a-b}{a+b}\left(\frac{1}{v_{n+1}^{m+1}}-\frac{1}{v_{n}^{m}}\right) .
$$

Since the constants $a$ and $b$ correspond to the lattice intervals of $m$ and $n$, respectively, eq.(1) can be also regarded as the discrete $\mathrm{KdV}$ equation on inhomogeneous lattice.

The non-autonomous version of discrete integrable systems on two-dimensional lattice have not been investigated well, although those on three-dimensional lattice, such as the Hirota-Miwa (discrete KP) equation or the discrete twodimensional Toda lattice equation, have been studied well together with their solutions [4, 5, 6, 7, 8]. Let us explain a reason taking eq.(1) as an example. The autonomous version eq.(2) can be transformed into so-called the bilinear equation by suitable dependent variable transformation. The bilinear equation is regarded as a reduction of the HirotaMiwa equation [9, 10], which is well-known to admit various types of exact solutions, such as soliton solutions[3, 9], rational solutions expressible in terms of the Schur functions [10, 11], or periodic solutions that are written in terms of the Riemann theta functions[12]. Therefore one can obtain solutions to the discrete KdV equation (2) by applying the reduction procedure to those for the Hirota-Miwa equation. Now, the Hirota-Miwa equation and its solutions can be generalized to non-autonomous case in a straightforward manner. However, it is shown that one cannot apply the reduction procedure to the non-autonomous Hirota-Miwa equation consistently. Moreover, eq. (1) cannot be put into bilinear equation by the procedure similar to the autonomous case because of the non-autonomous property. Therefore it was not clear how to construct solutions to the two-dimensional non-autonomous discrete integrable systems systematically.

In this article, we construct the Casorati determinant solution to the non-autonomous discrete KdV equation (1) by using the bilinear formalism. We present three different bilinearizations: The first one can be derived by the reduction of non-autonomous discrete KP hierarchy with a new technique. The second one is the bilinearization obtained by introducing certain auxiliary $\tau$ function which has a similar structure to the ones that appeared in the study of $R_{I}$ and $R_{I I}$ biorthogonal rational functions [13, 14]. The third bilinearization is through the use of non-autonomous potential discrete $\mathrm{KdV}$ equation. 
This article is organized as follows. In Section 2 we review the bilinearization of the discrete KdV equation (2), and discuss briefly why the similar calculation fails for the non-autonomous case. In Section 3 we discuss the bilinearizations of eq.(1) and construct the Casorati determinant solution. Finally, concluding remarks are given in Section 4.

\section{Bilinearization of the discrete $\mathrm{KdV}$ equation}

The discrete $\mathrm{KdV}$ equation (2) can be transformed to the bilinear equation

$$
\left(\frac{1}{a}+\frac{1}{b}\right) \tau_{n+1}^{m} \tau_{n-1}^{m+1}-\left(\frac{1}{a}-\frac{1}{b}\right) \tau_{n-1}^{m} \tau_{n+1}^{m+1}=\frac{2}{b} \tau_{n}^{m} \tau_{n}^{m+1}
$$

by the dependent variable transformation

$$
v_{n}^{m}=\frac{\tau_{n+1}^{m} \tau_{n}^{m+1}}{\tau_{n}^{m} \tau_{n+1}^{m+1}}
$$

In fact, substituting eq.(5) into eq.(2) we have

$$
\begin{aligned}
& \left(\frac{1}{a}+\frac{1}{b}\right) \tau_{n+1}^{m} \tau_{n-1}^{m+1} \tau_{n}^{m+2}-\left(\frac{1}{a}+\frac{1}{b}\right) \tau_{n-1}^{m+2} \tau_{n}^{m} \tau_{n+1}^{m+1} \\
= & \left(\frac{1}{a}-\frac{1}{b}\right) \tau_{n-1}^{m} \tau_{n+1}^{m+1} \tau_{n}^{m+2}-\left(\frac{1}{a}-\frac{1}{b}\right) \tau_{n+1}^{m+2} \tau_{n}^{m} \tau_{n-1}^{m+1} .
\end{aligned}
$$

Interchanging the second term of the left hand side and the first term of the right hand side, and dividing the both sides by $\tau_{n}^{m+2} \tau_{n}^{m} \tau_{n}^{m+1}$ we get

$$
\frac{\left(\frac{1}{a}+\frac{1}{b}\right) \tau_{n+1}^{m} \tau_{n-1}^{m+1}-\left(\frac{1}{a}-\frac{1}{b}\right) \tau_{n-1}^{m} \tau_{n+1}^{m+1}}{\tau_{n}^{m} \tau_{n}^{m+1}}=\frac{\left(\frac{1}{a}+\frac{1}{b}\right) \tau_{n+1}^{m+1} \tau_{n-1}^{m+2}-\left(\frac{1}{a}-\frac{1}{b}\right) \tau_{n-1}^{m+1} \tau_{n+1}^{m+2}}{\tau_{n}^{m+1} \tau_{n}^{m+2}} .
$$

Equation (7) can be decoupled as

$$
\left(\frac{1}{a}+\frac{1}{b}\right) \tau_{n+1}^{m} \tau_{n-1}^{m+1}-\left(\frac{1}{a}-\frac{1}{b}\right) \tau_{n-1}^{m} \tau_{n+1}^{m+1}=\alpha(n) \tau_{n}^{m} \tau_{n}^{m+1},
$$

since the right hand side of eq.(7) is obtained from the left hand side by shifting $m$ to $m+1$. Here $\alpha(n)$ is an arbitrary function in $n$, which can be absorbed by suitable gauge transformation on $\tau_{n}^{m}$. We obtain eq. (4) by choosing $\alpha(n)=\frac{2}{b}$ so that $\tau_{n}^{m}=1$ is a solution.

The bilinear equation (4) can be obtained by applying the reduction to the Hirota-Miwa equation

$$
\begin{aligned}
& a_{1}\left(a_{2}-a_{3}\right) \tau\left(l_{1}+1, l_{2}, l_{3}\right) \tau\left(l_{1}, l_{2}+1, l_{3}+1\right)+a_{2}\left(a_{3}-a_{1}\right) \tau\left(l_{1}, l_{2}+1, l_{3}\right) \tau\left(l_{1}+1, l_{2}, l_{3}+1\right) \\
+ & a_{3}\left(a_{1}-a_{2}\right) \tau\left(l_{1}, l_{2}, l_{3}+1\right) \tau\left(l_{1}+1, l_{2}+1, l_{3}\right)=0,
\end{aligned}
$$

where $a_{1}, a_{2}, a_{3}$ are arbitrary constants. In fact, imposing the condition

$$
\tau\left(l_{1}+1, l_{2}+1, l_{3}\right) \approx \tau\left(l_{1}, l_{2}, l_{3}\right),
$$

where $\approx$ means the equivalence up to gauge transformation, using eq.(10) to suppress the $l_{1}$ dependence and putting $a_{1}=-a_{2}$, eq. (9) yields

$$
\begin{aligned}
& -\left(a_{2}-a_{3}\right) \tau\left(l_{2}-1, l_{3}\right) \tau\left(l_{2}+1, l_{3}+1\right)+\left(a_{2}+a_{3}\right) \tau\left(l_{2}+1, l_{3}\right) \tau\left(l_{2}-1, l_{3}+1\right) \\
& -2 a_{3} \tau\left(l_{2}, l_{3}+1\right) \tau\left(l_{2}, l_{3}\right)=0
\end{aligned}
$$

which is equivalent to eq. (4) with $l_{2}=n, l_{3}=m, a_{2}=b, a_{3}=a$ and $\tau\left(l_{2}, l_{3}\right)=\tau_{n}^{m}$.

Now let us consider the non-autonomous case. We show that neither direct bilinearization nor reduction from the non-autonomous Hirota-Miwa equation work successfully for this case. First, substituting eq.(5) into eq.(1) and doing 
the same calculation as above, we arrive at the following equation

$$
\begin{aligned}
& \frac{\left(\frac{1}{a_{m}}+\frac{1}{b_{n}}\right) \tau_{n+1}^{m} \tau_{n-1}^{m+1}-\left(\frac{1}{a_{m}}-\frac{1}{b_{n-1}}\right) \tau_{n-1}^{m} \tau_{n+1}^{m+1}}{\tau_{n}^{m} \tau_{n}^{m+1}} \\
= & \frac{\left(\frac{1}{a_{m+1}}+\frac{1}{b_{n-1}}\right) \tau_{n+1}^{m+1} \tau_{n-1}^{m+2}-\left(\frac{1}{a_{m+1}}-\frac{1}{b_{n}}\right) \tau_{n-1}^{m+1} \tau_{n+1}^{m+2}}{\tau_{n}^{m+1} \tau_{n}^{m+2}},
\end{aligned}
$$

which cannot be decoupled into the bilinear equation because of $n$ dependence of the coefficients. Therefore naive bilinearization fails for the non-autonomous case.

Secondly, let us consider the reduction from the non-autonomous Hirota-Miwa equation [7, 8]

$$
\begin{aligned}
& a_{1}\left(l_{1}\right)\left(a_{2}\left(l_{2}\right)-a_{3}\left(l_{3}\right)\right) \tau\left(l_{1}+1, l_{2}, l_{3}\right) \tau\left(l_{1}, l_{2}+1, l_{3}+1\right) \\
+ & a_{2}\left(l_{2}\right)\left(a_{3}\left(l_{3}\right)-a_{1}\left(l_{1}\right)\right) \tau\left(l_{1}, l_{2}+1, l_{3}\right) \tau\left(l_{1}+1, l_{2}, l_{3}+1\right) \\
+ & a_{3}\left(l_{3}\right)\left(a_{1}\left(l_{1}\right)-a_{2}\left(l_{2}\right)\right) \tau\left(l_{1}, l_{2}, l_{3}+1\right) \tau\left(l_{1}+1, l_{2}+1, l_{3}\right)=0,
\end{aligned}
$$

where $a_{i}\left(l_{i}\right)(i=1,2,3)$ are arbitrary functions. Imposing the condition (10) on eq.(13) and suppressing the $l_{1}$ dependence, we obtain two different bilinear equations

$$
\begin{gathered}
a_{1}\left(l_{1}\right)\left(a_{2}\left(l_{2}\right)-a_{3}\left(l_{3}\right)\right) \tau\left(l_{2}-1, l_{3}\right) \tau\left(l_{2}+1, l_{3}+1\right) \\
+a_{2}\left(l_{2}\right)\left(a_{3}\left(l_{3}\right)-a_{1}\left(l_{1}\right)\right) \tau\left(l_{2}+1, l_{3}\right) \tau\left(l_{2}-1, l_{3}+1\right) \\
+a_{3}\left(l_{3}\right)\left(a_{1}\left(l_{1}\right)-a_{2}\left(l_{2}\right)\right) \tau\left(l_{2}, l_{3}+1\right) \tau\left(l_{2}, l_{3}\right)=0, \\
a_{1}\left(l_{1}-1\right)\left(a_{2}\left(l_{2}-1\right)-a_{3}\left(l_{3}\right)\right) \tau\left(l_{2}-1, l_{3}\right) \tau\left(l_{2}+1, l_{3}+1\right) \\
+a_{2}\left(l_{2}-1\right)\left(a_{3}\left(l_{3}\right)-a_{1}\left(l_{1}-1\right)\right) \tau\left(l_{2}+1, l_{3}\right) \tau\left(l_{2}-1, l_{3}+1\right) \\
+a_{3}\left(l_{3}\right)\left(a_{1}\left(l_{1}-1\right)-a_{2}\left(l_{2}-1\right)\right) \tau\left(l_{2}, l_{3}+1\right) \tau\left(l_{2}, l_{3}\right)=0 .
\end{gathered}
$$

Since those two equations should be equivalent, the coefficients must satisfy

$$
\begin{aligned}
& \frac{a_{1}\left(l_{1}\right)\left(a_{2}\left(l_{2}\right)-a_{3}\left(l_{3}\right)\right)}{a_{1}\left(l_{1}-1\right)\left(a_{2}\left(l_{2}-1\right)-a_{3}\left(l_{3}\right)\right)}=\frac{a_{2}\left(l_{2}\right)\left(a_{3}\left(l_{3}\right)-a_{1}\left(l_{1}\right)\right)}{a_{2}\left(l_{2}-1\right)\left(a_{3}\left(l_{3}\right)-a_{1}\left(l_{1}-1\right)\right)} \\
= & \frac{a_{3}\left(l_{3}\right)\left(a_{1}\left(l_{1}\right)-a_{2}\left(l_{2}\right)\right)}{a_{3}\left(l_{3}\right)\left(a_{1}\left(l_{1}-1\right)-a_{2}\left(l_{2}-1\right)\right)},
\end{aligned}
$$

which yields

$$
\begin{aligned}
& a_{3}\left(l_{3}\right)\left(\frac{1}{a_{1}\left(l_{1}\right) a_{2}\left(l_{2}-1\right)}-\frac{1}{a_{1}\left(l_{1}-1\right) a_{2}\left(l_{2}\right)}\right) \\
& +\left(\frac{1}{a_{1}\left(l_{1}-1\right)}-\frac{1}{a_{1}\left(l_{1}\right)}\right)-\left(\frac{1}{a_{2}\left(l_{2}-1\right)}-\frac{1}{a_{2}\left(l_{2}\right)}\right)=0 .
\end{aligned}
$$

Since this should hold for any $a\left(l_{3}\right)$ for all $l_{3}$, we deduce that $a\left(l_{1}\right)$ and $a\left(l_{2}\right)$ must be constants. This implies that it is not possible to impose the condition (10) on the non-autonomous Hirota-Miwa equation (13) consistently, unless it is reduced to the autonomous case.

\section{Bilinearizations of the non-autonomous discrete $\mathrm{KdV}$ equation}

\subsection{Reduction from the discrete KP hierarchy}

The non-autonomous discrete KP hierarchy in the bilinear form is expressed as [7, 3]

$$
\left|\begin{array}{cccccc}
1 & a_{i_{1}}\left(l_{i_{1}}\right) & a_{i_{1}}\left(l_{i_{1}}\right)^{2} & \ldots & a_{i_{1}}\left(l_{i_{1}}\right)^{m-2} & a_{i_{1}}\left(l_{i_{1}}\right)^{m-2} \tau_{i_{1}} \tau_{\hat{i_{1}}} \\
1 & a_{i_{2}}\left(l_{i_{2}}\right) & a_{i_{2}}\left(l_{i_{2}}\right)^{2} & \cdots & a_{i_{2}}\left(l_{i_{2}}\right)^{m-2} & a_{i_{2}}\left(l_{i_{2}}\right)^{m-2} \tau_{i_{2}} \tau_{\hat{i_{2}}} \\
\vdots & \vdots & \vdots & & \vdots & \vdots \\
1 & a_{i_{m}}\left(l_{i_{m}}\right) & a_{i_{m}}\left(l_{i_{m}}\right)^{2} & \ldots & a_{i_{m}}\left(l_{i_{m}}\right)^{m-2} & a_{i_{m}}\left(l_{i_{m}}\right)^{m-2} \tau_{i_{m}} \tau_{\hat{i_{m}}}
\end{array}\right|=0
$$


where $\left\{i_{1}, \ldots, i_{m}\right\} \subset\{1, \ldots, n\}, \tau_{i_{k}}$ and $\tau_{\hat{i}_{k}}(k=1, \ldots, m)$ are given by

$$
\begin{aligned}
\tau_{i_{k}} & =T_{i_{k}} \tau, \\
\tau_{\hat{i_{k}}} & =T_{i_{1}} T_{i_{2}} \cdots T_{i_{k-1}} T_{i_{k+1}} \cdots T_{i_{m}} \tau,
\end{aligned}
$$

respectively, $a_{v}\left(l_{v}\right)$ are arbitrary functions in $l_{v}$ for each $v$, and $n$ and $m$ are arbitrary integers satisfying $n \geq m \geq 3$. Here $T_{i}$ is the shift operator of $l_{i}$ defined by

$$
T_{i} \tau\left(l_{1}, l_{2}, \cdots, l_{n}\right)=\tau\left(l_{1}, l_{2}, \cdots, l_{i-1}, l_{i}+1, l_{i+1}, \cdots, l_{n}\right) .
$$

The simplest equation in the hierarchy $(m=3)$ is the non-autonomous Hirota-Miwa equation

$$
\begin{aligned}
& a_{i}\left(l_{i}\right)\left(a_{j}\left(l_{j}\right)-a_{k}\left(l_{k}\right)\right) \tau\left(l_{i}+1, l_{j}, l_{k}\right) \tau\left(l_{i}, l_{j}+1, l_{k}+1\right) \\
& +a_{j}\left(l_{j}\right)\left(a_{k}\left(l_{k}\right)-a_{i}\left(l_{i}\right)\right) \tau\left(l_{i}, l_{j}+1, l_{k}\right) \tau\left(l_{i}+1, l_{j}, l_{k}+1\right) \\
+ & a_{k}\left(l_{k}\right)\left(a_{i}\left(l_{i}\right)-a_{j}\left(l_{j}\right)\right) \tau\left(l_{i}, l_{j}, l_{k}+1\right) \tau\left(l_{i}+1, l_{j}+1, l_{k}\right)=0,
\end{aligned}
$$

where $\{i, j, k\} \subset\{1, \ldots, n\}$ and we suppressed other independent variables. The Casorati determinant solution to the hierarchy can be written as

$$
\tau\left(l_{1}, \cdots, l_{n}\right)=\left|\begin{array}{cccc}
\varphi_{1}^{(s)}\left(l_{1}, \cdots, l_{n}\right) & \varphi_{1}^{(s+1)}\left(l_{1}, \cdots, l_{n}\right) & \cdots & \varphi_{1}^{(s+N-1)}\left(l_{1}, \cdots, l_{n}\right) \\
\varphi_{2}^{(s)}\left(l_{1}, \cdots, l_{n}\right) & \varphi_{2}^{(s+1)}\left(l_{1}, \cdots, l_{n}\right) & \cdots & \varphi_{2}^{(s+N-1)}\left(l_{1}, \cdots, l_{n}\right) \\
\vdots & \vdots & \cdots & \vdots \\
\varphi_{N}^{(s)}\left(l_{1}, \cdots, l_{n}\right) & \varphi_{N}^{(s+1)}\left(l_{1}, \cdots, l_{n}\right) & \cdots & \varphi_{N}^{(s+N-1)}\left(l_{1}, \cdots, l_{n}\right)
\end{array}\right|,
$$

where $\varphi_{r}^{(s)}(r=1, \ldots, N)$ satisfy the linear equations

$$
\frac{\varphi_{r}^{(s)}\left(l_{1}, \cdots, l_{v}+1, \cdots, l_{n}\right)-\varphi_{r}^{(s)}\left(l_{1}, \cdots, l_{v}, \cdots, l_{n}\right)}{a_{v}\left(l_{v}\right)}=\varphi_{r}^{(s+1)}\left(l_{1}, \cdots, l_{v}, \cdots, l_{n}\right),
$$

for $v=1, \ldots, n$. For example, the $N$-soliton solution is obtained by choosing $\varphi_{r}^{(s)}$ as

$$
\varphi_{r}^{(s)}\left(l_{1}, \cdots, l_{n}\right)=\alpha_{r} p_{r}^{s} \prod_{\nu=1}^{n} \prod_{i=i_{\nu}}^{l_{\nu}-1}\left(1+a_{\nu}(i) p_{r}\right)+\beta_{r} q_{r}^{s} \prod_{\nu=1}^{n} \prod_{i=i_{\nu}}^{l_{\nu}-1}\left(1+a_{\nu}(i) q_{r}\right),
$$

where $\alpha_{r}, \beta_{r}, p_{r}, q_{r}(r=1, \ldots, N)$ are arbitrary constants.

Let us consider the reduction to the non-autonomous discrete $\mathrm{KdV}$ equation. The key idea is to specialize some of the independent variables to be autonomous by choosing the lattice intervals as constants and use them as auxiliary variables. Then one can use the autonomous variables for the reduction procedure to get the non-autonomous discrete $\mathrm{KdV}$ equation. We consider the four independent variables $k=l_{1}, l=l_{2}, m=l_{3}, n=l_{4}$ with the lattice intervals being $\delta=a_{1}(k), \epsilon=a_{2}(l), a_{m}=a_{3}(m), b_{n}=a_{4}(n)$, respectively. We note that $\delta$ and $\epsilon$ are constants, namely, $k$ and $l$ are autonomous variables. Then we have the following bilinear equations from eq.(22):

$$
\begin{aligned}
& \delta\left(a_{m}-b_{n}\right) \tau(k+1, l, m, n) \tau(k, l, m+1, n+1) \\
+ & a_{m}\left(b_{n}-\delta\right) \tau(k, l, m+1, n) \tau(k+1, l, m, n+1) \\
+ & b_{n}\left(\delta-a_{m}\right) \tau(k, l, m, n+1) \tau(k+1, l, m+1, n)=0, \\
& \epsilon\left(a_{m}-b_{n}\right) \tau(k, l+1, m, n) \tau(k, l, m+1, n+1) \\
+ & a_{m}\left(b_{n}-\epsilon\right) \tau(k, l, m+1, n) \tau(k, l+1, m, n+1) \\
+ & b_{n}\left(\epsilon-a_{m}\right) \tau(k, l, m, n+1) \tau(k, l+1, m+1, n)=0 .
\end{aligned}
$$

We impose the condition

$$
\tau(k+1, l+1, m, n) \approx \tau(k, l, m, n) .
$$

This is achieved by imposing the condition on $\varphi_{r}^{(s)}(r=1, \ldots, N)$ as

$$
\varphi_{r}^{(s)}(k+1, l+1, m, n) \approx \varphi_{r}^{(s)}(k, l, m, n) .
$$


For the case of soliton solutions, $\varphi_{r}^{(s)}(k, l, m, n)$ is expressed as

$$
\begin{aligned}
\varphi_{r}^{(s)}(k, l, m, n) & =\alpha_{r} p_{r}^{s}\left(1+\delta p_{r}\right)^{k}\left(1+\epsilon p_{r}\right)^{l} \prod_{i=m_{0}}^{m-1}\left(1+a_{i} p_{r}\right) \prod_{j=n_{0}}^{n-1}\left(1+b_{j} p_{r}\right) \\
& +\beta_{r} q_{r}^{s}\left(1+\delta q_{r}\right)^{k}\left(1+\epsilon q_{r}\right)^{l} \prod_{i=m_{0}}^{m-1}\left(1+a_{i} q_{r}\right) \prod_{j=n_{0}}^{n-1}\left(1+b_{j} q_{r}\right) .
\end{aligned}
$$

In order to satisfy eq.(29), one may take

$$
q_{r}=-p_{r}, \quad \delta=-\epsilon
$$

so that

$$
\begin{aligned}
& \varphi_{r}^{(s)}(k+1, l+1, m, n)=\left(1-\epsilon^{2} p_{r}^{2}\right) \varphi_{r}^{(s)}(k, l, m, n), \\
& \tau(k+1, l+1, m, n)=\prod_{r=1}^{N}\left(1-\epsilon^{2} p_{r}^{2}\right) \tau(k, l, m, n) .
\end{aligned}
$$

Then, suppressing the $k$-dependence by using eq.(28), the bilinear equations (26) and (27) are reduced to

$$
\begin{aligned}
& -\epsilon\left(a_{m}-b_{n}\right) \tau(l, m, n) \tau(l+1, m+1, n+1)+a_{m}\left(b_{n}+\epsilon\right) \tau(l+1, m+1, n) \tau(l, m, n+1) \\
& -b_{n}\left(\epsilon+a_{m}\right) \tau(l+1, m, n+1) \tau(l, m+1, n)=0 \\
& \epsilon\left(a_{m}-b_{n}\right) \tau(l+1, m, n) \tau(l, m+1, n+1)+a_{m}\left(b_{n}-\epsilon\right) \tau(l, m+1, n) \tau(l+1, m, n+1) \\
& +b_{n}\left(\epsilon-a_{m}\right) \tau(l, m, n+1) \tau(l+1, m+1, n)=0
\end{aligned}
$$

respectively. By putting

$$
\tau_{n}^{m}=\tau(l, m, n), \quad \sigma_{n}^{m}=\tau(l+1, m, n),
$$

the above bilinear equations are rewritten as

$$
\begin{aligned}
& -\epsilon\left(a_{m}-b_{n}\right) \tau_{n}^{m} \sigma_{n+1}^{m+1}+a_{m}\left(b_{n}+\epsilon\right) \tau_{n+1}^{m} \sigma_{n}^{m+1}-b_{n}\left(\epsilon+a_{m}\right) \tau_{n}^{m+1} \sigma_{n+1}^{m}=0, \\
& \epsilon\left(a_{m}-b_{n}\right) \sigma_{n}^{m} \tau_{n+1}^{m+1}+a_{m}\left(b_{n}-\epsilon\right) \tau_{n}^{m+1} \sigma_{n+1}^{m}+b_{n}\left(\epsilon-a_{m}\right) \tau_{n+1}^{m} \sigma_{n}^{m+1}=0,
\end{aligned}
$$

respectively. Equations (33) and (34) can be regarded as a bilinearization of the non-autonomous discrete KdV equation (1). In fact, introducing the variables $\Psi_{n}^{m}$ and $v_{n}^{m}$ by

$$
\begin{aligned}
& \Psi_{n}^{m}=\frac{\sigma_{n}^{m}}{\tau_{n}^{m}}, \\
& v_{n}^{m}=\frac{\tau_{n+1}^{m} \tau_{n}^{m+1}}{\tau_{n}^{m} \tau_{n+1}^{m+1}},
\end{aligned}
$$

we obtain

$$
\begin{aligned}
& \left(\frac{1}{b_{n}}-\frac{1}{a_{m}}\right) \frac{1}{v_{n}^{m}} \Psi_{n+1}^{m+1}-\left(\frac{1}{\epsilon}+\frac{1}{b_{n}}\right) \Psi_{n}^{m+1}+\left(\frac{1}{\epsilon}+\frac{1}{a_{m}}\right) \Psi_{n+1}^{m}=0, \\
& \left(\frac{1}{b_{n}}-\frac{1}{a_{m}}\right) \frac{1}{v_{n}^{m}} \Psi_{n}^{m}+\left(\frac{1}{\epsilon}-\frac{1}{b_{n}}\right) \Psi_{n+1}^{m}+\left(\frac{1}{a_{m}}-\frac{1}{\epsilon}\right) \Psi_{n}^{m+1}=0,
\end{aligned}
$$

which are regarded as the auxiliary linear problem for the non-autonomous discrete $\mathrm{KdV}$ equation. Eliminating $\Psi_{m}^{n}$ by considering the compatibility condition we obtain the non-autonomous discrete $\mathrm{KdV}$ equation (1). The $N$-soliton solution is given by

$$
\tau_{n}^{m}=\left|\begin{array}{cccc}
\varphi_{1}^{(s)}(m, n) & \varphi_{1}^{(s+1)}(m, n) & \cdots & \varphi_{1}^{(s+N-1)}(m, n) \\
\varphi_{2}^{(s)}(m, n) & \varphi_{2}^{(s+1)}(m, n) & \cdots & \varphi_{2}^{(s+N-1)}(m, n) \\
\vdots & \vdots & \cdots & \vdots \\
\varphi_{N}^{(s)}(m, n) & \varphi_{N}^{(s+1)}(m, n) & \cdots & \varphi_{N}^{(s+N-1)}(m, n)
\end{array}\right|
$$




$$
\varphi_{r}^{(s)}(m, n)=\alpha_{r} p_{r}^{s} \prod_{i=m_{0}}^{m-1}\left(1+a_{i} p_{r}\right) \prod_{j=n_{0}}^{n-1}\left(1+b_{j} p_{r}\right)+\beta_{r}\left(-p_{r}\right)^{s} \prod_{i=m_{0}}^{m-1}\left(1-a_{i} p_{r}\right) \prod_{j=n_{0}}^{n-1}\left(1-b_{j} p_{r}\right) .
$$

We remark that we obtain the non-autonomous potential discrete modified KdV equation for $\Psi_{n}^{m}$ by eliminating $v_{n}^{m}$ from eqs. (36) and (37).

\subsection{Alternate bilinearization}

There is another interesting bilinearization to the non-autonomous discrete KdV equation (1). Let us consider the following bilinear equations,

$$
\begin{aligned}
& b_{n}\left(a_{m-1}+a_{m}\right) \kappa_{n+1}^{m} \tau_{n}^{m}-a_{m-1}\left(a_{m}+b_{n}\right) \tau_{n+1}^{m-1} \tau_{n}^{m+1}+a_{m}\left(a_{m-1}-b_{n}\right) \tau_{n+1}^{m+1} \tau_{n}^{m-1}=0, \\
& b_{n}\left(a_{m-1}-a_{m}\right) \tau_{n}^{m+1} \tau_{n+1}^{m-1}-a_{m}\left(a_{m-1}-b_{n}\right) \tau_{n+1}^{m} \kappa_{n}^{m}+a_{m-1}\left(a_{m}-b_{n}\right) \tau_{n}^{m} \kappa_{n+1}^{m}=0 .
\end{aligned}
$$

We obtain eq.(1) by introducing $v_{n}^{m}$ by eq. (35b) and eliminating $\kappa_{n}^{m}$. The Casorati determinant solution is given by

$$
\kappa_{n}^{m}=\left|\begin{array}{cccc}
\psi_{1}^{(s)}(m, n) & \psi_{1}^{(s+1)}(m, n) & \cdots & \psi_{1}^{(s+N-1)}(m, n) \\
\psi_{2}^{(s)}(m, n) & \psi_{2}^{(s+1)}(m, n) & \cdots & \psi_{2}^{(s+N-1)}(m, n) \\
\vdots & \vdots & \cdots & \vdots \\
\psi_{N}^{(s)}(m, n) & \psi_{N}^{(s+1)}(m, n) & \cdots & \psi_{N}^{(s+N-1)}(m, n)
\end{array}\right|
$$

where

$$
\begin{aligned}
\psi_{r}^{(s)}(m, n) & =\lambda_{r} p_{r}^{s}\left(1+a_{m} p_{r}\right) \prod_{i=m_{0}}^{m-2}\left(1+a_{i} p_{r}\right) \prod_{j=n_{0}}^{n-1}\left(1+b_{j} p_{r}\right) \\
& +\mu_{r}\left(-p_{r}\right)^{s}\left(1-a_{m} p_{r}\right) \prod_{i=l_{0}}^{m-2}\left(1-b_{i} p_{r}\right) \prod_{j=n_{0}}^{n-1}\left(1-b_{j} p_{r}\right),
\end{aligned}
$$

$\lambda_{r}, \mu_{r}$ are arbitrary constants $(r=1, \ldots, N)$ and $\tau_{n}^{m}$ is given by eqs.(38) and (39).

We note that in the autonomous case, $\kappa_{n}^{m}$ reduces to $\tau_{n}^{m}$, the bilinear equation (40) yields eq.(4), and eq.(41) becomes trivial, respectively. Secondly, because of the symmetry with respect to $m, n$ in eqs.(1) and (35b), the following bilinearization is also possible:

$$
\begin{aligned}
& a_{m}\left(b_{n-1}+b_{n}\right) \theta_{n}^{m+1} \tau_{n}^{m}-b_{n-1}\left(b_{n}+a_{m}\right) \tau_{n-1}^{m+1} \tau_{n+1}^{m}+b_{n}\left(b_{n-1}-a_{m}\right) \tau_{n+1}^{m+1} \tau_{n-1}^{m}=0, \\
& a_{m}\left(b_{n-1}-b_{n}\right) \tau_{n+1}^{m} \tau_{n-1}^{m+1}-b_{n}\left(b_{n-1}-a_{m}\right) \tau_{n}^{m+1} \theta_{n}^{m}+b_{n-1}\left(b_{n}-a_{m}\right) \tau_{n}^{m} \theta_{n}^{m+1}=0,
\end{aligned}
$$

whose solution is expressed as

$$
\begin{gathered}
\theta_{n}^{m}=\left|\begin{array}{cccc}
\phi_{1}^{(s)}(m, n) & \phi_{1}^{(s+1)}(m, n) & \cdots & \phi_{1}^{(s+N-1)}(m, n) \\
\phi_{2}^{(s)}(m, n) & \phi_{2}^{(s+1)}(m, n) & \cdots & \phi_{2}^{(s+N-1)}(m, n) \\
\vdots & \vdots & \cdots & \vdots \\
\phi_{N}^{(s)}(m, n) & \phi_{N}^{(s+1)}(m, n) & \cdots & \phi_{N}^{(s+N-1)}(m, n)
\end{array}\right|, \\
\phi_{r}^{(s)}(m, n)=\lambda_{r} p_{r}^{s}\left(1+b_{n} p_{r}\right) \prod_{i=m_{0}}^{m-1}\left(1+a_{i} p_{r}\right) \prod_{j=n_{0}}^{n-2}\left(1+b_{j} p_{r}\right) \\
+\mu_{r}\left(-p_{r}\right)^{s}\left(1-b_{n} p_{r}\right) \prod_{i=l_{0}}^{m-1}\left(1-a_{i} p_{r}\right) \prod_{j=n_{0}}^{n-2}\left(1-b_{j} p_{r}\right) .
\end{gathered}
$$

We also remark that the similar structure in the above auxiliary $\tau$ functions has appeared in the study of $R_{I}$ and $R_{I I}$ biorthogonal functions [13, 14]. Also, similar soliton type solution has been constructed for the non-autonomous discrete-time Toda lattice equation[15]. 
We can show that $\tau_{n}^{m}$ and $\kappa_{n}^{m}$ satisfy eqs.(40) and (41) by the technique similar to that was used in refs. [13, 14, 15]. Namely, by using the linear relations among $\varphi_{r}^{(s)}$ and $\psi_{r}^{(s)}$, we first construct such difference formulas that express the determinants whose columns are appropriately shifted by $\tau_{n}^{m}$ or $\kappa_{n}^{m}$. Then eqs. (41) and (44) are derived from Plücker relations which are quadratic identities of determinants whose columns are shifted.

From eqs. (39) and (43), we see that $\varphi_{r}^{(s)}$ and $\psi_{r}^{(s)}$ satisfy

$$
\begin{aligned}
& \varphi_{r}^{(s)}(m+1, n)-\varphi_{r}^{(s)}(m, n)=a_{m} \varphi_{r}^{(s+1)}(m, n), \\
& \varphi_{r}^{(s)}(m-1, n)+a_{m} \varphi_{r}^{(s+1)}(m-1, n)=\psi_{r}^{(s)}(m, n), \\
& \psi_{r}^{(s)}(m, n)-a_{m} \psi_{r}^{(s+1)}(m, n)=\left(1-a_{m}^{2} p_{r}^{2}\right) \varphi_{r}^{(s)}(m-1, n), \\
& \varphi_{r}^{(s)}(m, n+1)-\varphi_{r}^{(s)}(m, n)=b_{n} \varphi_{r}^{(s+1)}(m, n) .
\end{aligned}
$$

We introduce a notation

$$
\tau_{n}^{m}=|0,1, \cdots, N-2, N-1|
$$

where " $k$ " denotes the column vector

$$
k_{n}^{m}=\left(\begin{array}{c}
\varphi_{1}^{(s+k)}(m, n) \\
\vdots \\
\varphi_{N}^{(s+k)}(m, n)
\end{array}\right) .
$$

Then the following difference formulas are derived from eqs.(48)-(51) by the similar calculations to those given in ref.[15]:

$$
\begin{aligned}
-a_{m-1} \tau_{n}^{m-1} & =\left|0,1, \cdots, N-3, N-2, N-2_{m-1}\right|, \\
-b_{n-1} \tau_{n-1}^{m} & =\left|0,1, \cdots, N-3, N-2, N-2_{n-1}\right|, \\
A(m)^{-1} a_{m} \tau_{n}^{m+1} & =\left|0,1, \cdots, N-3, N-2, \widetilde{N-2_{m+1}}\right|, \\
-A(m)^{-1}\left(a_{m-1}+a_{m}\right) \sigma_{n}^{m} & =\left|0,1, \cdots, N-3, \widetilde{N-2_{m+1}}, N-2_{m-1}\right|, \\
-\left(a_{m-1}-b_{n-1}\right) \tau_{n-1}^{m-1} & =\left|0,1, \cdots, N-3, N-2_{n-1}, N-2_{m-1}\right|, \\
A(m)^{-1}\left(a_{m}+b_{n-1}\right) \tau_{n-1}^{m+1} & =\left|0,1, \cdots, N-3, N-2_{n-1}, \widetilde{N-2_{m+1}}\right|,
\end{aligned}
$$

where

$$
\begin{gathered}
\widetilde{k}_{m+1}=\left(\begin{array}{c}
A_{1}(m)^{-1} \varphi_{1}^{(s+k)}(m+1, n) \\
\vdots \\
A_{N}(m)^{-1} \varphi_{N}^{(s+k)}(m+1, n)
\end{array}\right), \\
A_{r}(m)=1-a_{m}^{2} p_{r}^{2} \quad(r=1, \ldots, N), \quad A(m)=\prod_{r=1}^{N}\left(1-a_{m}^{2} p_{r}^{2}\right) .
\end{gathered}
$$

We give the proof of the above formulas in the appendix. Applying the the difference formulas to the Plücker relation

$$
\begin{aligned}
0 & =\left|0, \cdots, N-3, N-2, N-2_{n-1}\right| \times\left|0, \cdots, N-3, N-2_{m-1}, \widetilde{N-} 2_{m+1}\right| \\
& -\left|0, \cdots, N-3, N-2, N-2_{m-1}\right| \times\left|0, \cdots, N-3, N-2_{n-1}, \widetilde{N-} 2_{m+1}\right| \\
& +\left|0, \cdots, N-3, N-2, \widetilde{N-2} 2_{m+1}\right| \times\left|0, \cdots, N-3, N-2_{n-1}, N-2_{m-1}\right|,
\end{aligned}
$$

we obtain the bilinear equation (41). Equation (44) is derived by applying the difference formulas (54), (55), (58) and

$$
\begin{aligned}
&-a_{m-2} \sigma_{n}^{m-1}=\left|0,1, \cdots, N-3, N-2, \widehat{N-2} 2_{m-1}\right|, \\
&-\left(a_{m-2}-a_{m-1}\right) \tau_{n}^{m-2}=\left|0,1, \cdots, N-3, N-2_{m-1}, \widehat{N-} 2_{m-1}\right|, \\
&-\left(a_{m-2}-b_{n-1}\right) \sigma_{n-1}^{m-1}=\left|0,1, \cdots, N-3, N-2_{n-1}, \widehat{N-2_{m-1}}\right|,
\end{aligned}
$$


where

$$
\widehat{k}_{m-1}=\left(\begin{array}{c}
\psi_{1}^{(s+k)}(m-1, n) \\
\vdots \\
\psi_{N}^{(s+k)}(m-1, n)
\end{array}\right),
$$

to the Plücker relation,

$$
\begin{aligned}
0 & =\left|0, \cdots, N-3, N-2, N-2_{n-1}\right| \times\left|0, \cdots, N-3, N-2_{m-1}, \widehat{N-2_{m-1}}\right| \\
& -\left|0, \cdots, N-3, N-2, N-2_{m-1}\right| \times\left|0, \cdots, N-3, N-2_{n-1}, \widehat{N-2_{m-1}}\right| \\
& +\left|0, \cdots, N-3, N-2, \widehat{N-2} 2_{m-1}\right| \times\left|0, \cdots, N-3, N-2_{n-1}, N-2_{m-1}\right| .
\end{aligned}
$$

\subsection{Reduction from the KP hierarchy through the potential form}

In this section, we consider the following difference equation

$$
u_{n+1}^{m+1}-u_{n}^{m}=\left(\frac{1}{a_{m}^{2}}-\frac{1}{b_{n}^{2}}\right) \frac{1}{u_{n}^{m+1}-u_{n+1}^{m}},
$$

which is closely related to eq.(1) as

$$
\left(\frac{1}{a_{m}}-\frac{1}{b_{n}}\right) \frac{1}{v_{n}^{m}}=u_{n+1}^{m}-u_{n}^{m+1}
$$

The autonomous version of eq. (68) is known as the potential discrete $\mathrm{KdV}$ equation[16]. We call eq.(68) the nonautonomous potential discrete $\mathrm{KdV}$ equation. Casorati determinant solution to eq. (68) is given by

$$
u_{n}^{m}=\frac{\rho_{n}^{m}}{\tau_{n}^{m}}-\sum_{i=m_{0}}^{m-1} \frac{1}{a_{i}}-\sum_{j=n_{0}}^{n-1} \frac{1}{b_{j}},
$$

where

$$
\rho_{n}^{m}=\left|\begin{array}{cccc}
\varphi_{1}^{(s)}(m, n) & \cdots & \varphi_{1}^{(s+N-2)}(m, n) & \varphi_{1}^{(s+N)}(m, n) \\
\varphi_{2}^{(s)}(m, n) & \cdots & \varphi_{2}^{(s+N-2)}(m, n) & \varphi_{2}^{(s+N)}(m, n) \\
\vdots & \vdots & \cdots & \vdots \\
\varphi_{N}^{(s)}(m, n) & \cdots & \varphi_{N}^{(s+N-2)}(m, n) & \varphi_{N}^{(s+N)}(m, n)
\end{array}\right|,
$$

and $\varphi_{r}^{(s)}(m, n)(r=1, \ldots, N), \tau_{n}^{m}$ are defined by eqs.(39) and (38), respectively.

Equation (68) is derived from the following bilinear equations for $\rho_{n}^{m}$ and $\tau_{n}^{m}$

$$
\begin{aligned}
& \rho_{n}^{m+1} \tau_{n+1}^{m}-\rho_{n+1}^{m} \tau_{n}^{m+1}=\left(\frac{1}{a_{m}}-\frac{1}{b_{n}}\right)\left(\tau_{n}^{m+1} \tau_{n+1}^{m}-\tau_{n+1}^{m+1} \tau_{n}^{m}\right), \\
& \rho_{n+1}^{m+1} \tau_{n}^{m}-\rho_{n}^{m} \tau_{n+1}^{m+1}=\left(\frac{1}{a_{m}}+\frac{1}{b_{n}}\right)\left(\tau_{n+1}^{m+1} \tau_{n}^{m}-\tau_{n}^{m+1} \tau_{n+1}^{m}\right),
\end{aligned}
$$

through the dependent variable transformation (70). In particular, eq.(69) also follows from eq.(72). Therefore, we may regard eqs. (72) and (73) as yet another bilinearization of the non-autonomous discrete KdV equation (1).

We can show that $\rho_{n}^{m}$ and $\tau_{n}^{m}$ satisfy eqs. (72) and (73) as follows. Applying the difference formulas (54), (55), (58) and

$$
\begin{aligned}
-\left(a_{m-1} \rho_{n}^{m-1}+\tau_{n}^{m-1}\right) & =\left|0,1, \cdots, N-3, N-1, N-2_{m-1}\right|, \\
-\left(b_{n-1} \rho_{n-1}^{m}+\tau_{n-1}^{m}\right) & =\left|0,1, \cdots, N-3, N-1, N-2_{n-1}\right|,
\end{aligned}
$$

to the Plücker relation

$$
\begin{aligned}
0 & =|0, \cdots, N-3, N-2, N-1| \times\left|0, \cdots, N-3, N-2_{n-1}, N-2_{m-1}\right| \\
& -\left|0, \cdots, N-3, N-2, N-2_{n-1}\right| \times\left|0, \cdots, N-3, N-1, N-2_{m-1}\right| \\
& +\left|0, \cdots, N-3, N-2, N-2_{m-1}\right| \times\left|0, \cdots, N-3, N-1, N-2_{n-1}\right|,
\end{aligned}
$$


we have eq. (72). Similarly, we obtain eq.(73) by applying the formulas (55), (56), (59), (75) and

$$
A(m)^{-1}\left(a_{m} \rho_{n}^{m+1}-\tau_{n}^{m+1}\right)=\left|0, \cdots, N-3, N-1, \widetilde{N-2}{ }_{m+1}\right|,
$$

to the Plücker relation

$$
\begin{aligned}
0 & =|0, \cdots, N-3, N-2, N-1| \times\left|0, \cdots, N-3, N-2_{n-1}, \widetilde{N-} 2_{m+1}\right| \\
& -\left|0, \cdots, N-3, N-2, N-2_{n-1}\right| \times\left|0, \cdots, N-3, N-1, \widetilde{N-} 2_{m+1}\right| \\
& +\left|0, \cdots, N-3, N-2, \widetilde{N-} 2_{m+1}\right| \times\left|0, \cdots, N-3, N-1, N-2_{n-1}\right| .
\end{aligned}
$$

We finally remark that if we introduce the continuous independent variables $t_{1}, t_{3}, \cdots$ through $\varphi_{r}^{(s)}(m, n)$ as

$$
\begin{aligned}
\varphi_{r}^{(s)}(m, n) & =\alpha_{r} p_{r}^{s} \prod_{i=m_{0}}^{m-1}\left(1+a_{i} p_{r}\right) \prod_{j=n_{0}}^{n-1}\left(1+b_{j} p_{r}\right) e^{p_{r} t_{1}+p_{r}^{3} t_{3}+\cdots} \\
& +\beta_{r}\left(-p_{r}\right)^{s} \prod_{i=m_{0}}^{m-1}\left(1-a_{i} p_{r}\right) \prod_{j=n_{0}}^{n-1}\left(1-b_{j} p_{r}\right) e^{-p_{r} t_{1}-p_{r}^{3} t_{3}+\cdots}
\end{aligned}
$$

then $\tau_{n}^{m}$ becomes the $\tau$ function of the $\mathrm{KdV}$ hierarchy. In this case, $\rho_{n}^{m}$ and $u_{n}^{m}$ can be expressed as

$$
\rho_{n}^{m}=\frac{\partial \tau_{n}^{m}}{\partial t_{1}}, \quad u_{n}^{m}=\frac{\partial}{\partial t_{1}} \log \tau_{n}^{m}
$$

respectively, and $u_{n}^{m}$ satisfies the potential $\mathrm{KdV}$ equation

$$
\frac{\partial u_{n}^{m}}{\partial t_{3}}-\frac{3}{2}\left(\frac{\partial u_{n}^{m}}{\partial t_{1}}\right)^{2}-\frac{1}{4} \frac{\partial^{3} u_{n}^{m}}{\partial t_{1}^{3}}=0 .
$$

This is consistent with the fact that (autonomous version of) eq. (68) is derived as the Bäcklund transformation of the potential KdV equation[16].

\section{Concluding remarks}

In this article, we have considered the bilinearization of the non-autonomous discrete $\mathrm{KdV}$ equation and constructed Casorati determinant solution. We have presented three different bilinearizations, each of which has different origin. Although we have constructed only Casorati determinant solution, namely, soliton type solution, it might not be difficult to discuss other types of solutions, such as rational solutions or periodic solutions, based on the bilinear equations that have been obtained in this article. Also, we expect that other non-autonomous discrete integrable systems on two-dimensional lattice can be investigated in similar manner.

As was mentioned in Section 3.2, the $\tau$ functions in the second bilinearization resemble those in the theory of $R_{I}$ and $R_{I I}$ biorthogonal functions, but the explicit relation is not clear yet. It might be an intriguing problem to study underlying structure of the second bilinearization.

Finally, recently Takahashi and Hirota have succeeded in constructing the soliton solutions of the ultradiscrete $\mathrm{KdV}$ equation in permanent form[17]. It might be an interesting problem to investigate the permanent type solutions for the non-autonomous case.

\section{Acknowledgments}

The authors would like to express their sincere gratitude to Professor N. Matsuura for stimulating discussions and fruitful informations which motivated this work. They are also grateful to Professor A. Nakayashiki for valuable discussions and useful comments. 


\section{A Proof of difference formulas}

In the appendix, we give the proof of the difference formulas of $\tau$ functions which have been used in the derivation of bilinear equations from the Plücker relations for completeness. For later convenience, we first prepare the following two equations for $\varphi_{r}^{(s)}$ and $\psi_{r}^{(s)}$ which are derived from eqs. (48)-(50):

$$
\begin{aligned}
& \psi_{r}^{(s)}(m, n)+a_{m-1} \psi_{r}^{(s+1)}(m, n)=\varphi_{r}^{(s)}(m+1, n), \\
& \varphi_{r}^{(s)}(m+1, n)-a_{m} \varphi_{r}^{(s+1)}(m+1, n)=A_{r}(m) \varphi_{r}^{(s)}(m, n) .
\end{aligned}
$$

Equations (54) and (55) We have

$$
\tau_{n}^{m-1}=\left|0_{m-1}, 1_{m-1}, \cdots, N-2_{m-1}, N-1_{m-1}\right| .
$$

Adding the $(i+1)$-th column multiplied by $a_{m-1}$ to the $i$-th column for $i=1,2, \ldots, N-1$ and using eq. (48), we have

$$
\tau_{n}^{m-1}=\left|0,1, \cdots, N-2, N-1_{m-1}\right| .
$$

Multiplying $a_{m-1}$ to the $N$-th column and using eq. (48) we obtain

$$
\begin{aligned}
a_{m-1} \tau_{n}^{m-1} & =\left|0,1, \cdots, N-2, a_{m-1} \times(N-1)_{m-1}\right| \\
& =\left|0,1, \cdots, N-2,(N-2)_{m}-(N-2)_{m-1}\right| \\
& =-\left|0,1, \cdots, N-2, N-2_{m-1}\right|,
\end{aligned}
$$

which is eq.(54). Equation (55) can be shown in a similar manner by shifting $n$ and using eq.(51).

Equation (56) We have

$$
\tau_{n}^{m+1}=\left|0_{m+1}, 1_{m+1}, \cdots, N-2_{m+1}, N-1_{m+1}\right| .
$$

Adding the $(i+1)$-th column multiplied by $-a_{m}$ to the $i$-th column for $i=1,2, \ldots, N-1$ and using eq. (83), we have

$$
\tau_{n}^{m+1}=\left|\overline{0}, \overline{1}, \cdots, \overline{N-2}, N-1_{m+1}\right|, \quad \bar{k}=\left(\begin{array}{c}
A_{1}(m) \varphi_{1}^{(s)}(m, n) \\
\vdots \\
A_{N}(m) \varphi_{N}^{(s)}(m, n)
\end{array}\right) .
$$

Multiplying $a_{m}$ to the $N$-th column and using eq.(83) we have

$$
\begin{aligned}
a_{m} \tau_{n}^{m+1} & =\left|\overline{0}, \overline{1}, \cdots, \overline{N-2}, a_{m} \times(N-1)_{m+1}\right| \\
& =\left|\overline{0}, \overline{1}, \cdots, \overline{N-2}, N-2_{m+1}\right| \\
& =A(m) \times\left|0,1, \cdots, N-2, \widetilde{N-} 2_{m+1}\right|,
\end{aligned}
$$

which is eq. (56).

Equation (57) We have

$$
\sigma_{n}^{m}=|\widehat{0}, \widehat{1}, \cdots, \widehat{N-1}|,
$$

which is rewritten by using eq. (82) from the first column to the $N$-th column as

$$
\sigma_{n}^{m}=\left|0_{m+1}, 1_{m+1}, \cdots, N-2_{m+1}, \widehat{N-1} 1_{m}\right| .
$$

Now, from eqs.(50) and (82) we have

$$
A_{r}(m) \varphi_{r}^{(s)}(m-1, n)=-\left(a_{m}+a_{m-1}\right) \psi_{r}^{(s+1)}(m, n)+\varphi_{r}^{(s)}(m+1, n) .
$$

Applying eq. (89) to the $N$-th column of the right hand side of eq. (88) we obtain

$$
\begin{aligned}
\left(a_{m}+a_{m-1}\right) \sigma_{n}^{m} & =-\left|0_{m+1}, \cdots, N-2_{m+1}, \overline{N-2}_{m-1}\right| \\
& =-\left|\overline{0}_{m}, \cdots, \overline{N-3}_{m}, N-2_{m+1}, \overline{N-2}_{m-1}\right| \\
& =-A(m) \times\left|0_{m}, \cdots, N-3_{m}, \widetilde{N-2} 2_{m+1}, N-2_{m-1}\right|,
\end{aligned}
$$

which is eq.57). 
Equation (58) Shifting $n$ in eq.(54) we have

$$
-a_{m-1} \tau_{n-1}^{m-1}=\left|0_{n-1}, 1_{n-1}, \cdots, N-3_{n-1}, N-2_{n-1}, N-2_{\substack{m-1 \\ n-1}}\right| .
$$

Eliminating $\varphi_{r}^{(s+1)}(m-1, n-1)$ from eqs. (48) ${ }_{n-1}^{m-1}$ and ${ }_{51}^{m-1}{ }_{n-1}^{m}$ we get

$$
\left(a_{m-1}-b_{n-1}\right) \varphi_{r}^{(s)}(m-1, n-1)=a_{m-1} \varphi_{r}^{(s)}(m-1, n)-b_{n-1} \varphi_{r}^{(s)}(m, n-1) .
$$

Using eq.91) to the $N$-th column of the right hand side of eq.90), we have

$$
-a_{m-1}\left(a_{m-1}-b_{n-1}\right) \tau_{n-1}^{m-1}=a_{m-1}\left|0_{n-1}, 1_{n-1}, \cdots, N-3_{n-1}, N-2_{n-1}, N-2_{m-1}\right| .
$$

Adding $(i+1)$-th column multiplied by $a_{m-1}$ to $i$-th column for $i=1,2, \ldots, N-1$ and using eq. (48), we obtain

$$
-\left(a_{m-1}-b_{n-1}\right) \tau_{n-1}^{m-1}=\left|0,1, \cdots, N-3, N-2_{n-1}, N-2_{m-1}\right|,
$$

which is eq.58).

Equation (59) Shifting $n$ in eq.(56) we have

$$
\begin{aligned}
A(m)^{-1} a_{m} \tau_{n-1}^{m+1} & =\left|0_{n-1}, 1_{n-1}, \cdots, N-3_{n-1}, N-2_{n-1}, \widetilde{N-2^{m+1}}\right| \\
& =\left|0,1, \cdots, N-3, N-2_{n-1}, \widetilde{N-2_{n+1}^{m+1}}\right|,
\end{aligned}
$$

or

$$
a_{m} \tau_{n-1}^{m+1}=\left|\overline{0}, \overline{1}, \cdots, \overline{N-3}, \overline{N-2}{ }_{n-1}, N-2_{\substack{m+1 \\ n-1}}\right| .
$$

Eliminating $\varphi_{r}^{(s+1)}(m+1, n-1)$ from eqs. $(83)_{n-1}$ and $(51)_{n+1}^{m+1}$ we get

$$
b_{n-1} A_{r}(m) \varphi_{r}^{(s)}(m, n-1)+a_{m} \varphi_{r}^{(s)}(m+1, n)=\left(a_{m}+b_{n-1}\right) \varphi_{r}^{(s)}(m+1, n-1) .
$$

Applying eq.(93) to the $N$-th column of the right hand side of eq.(92), we have

$$
a_{m}\left(a_{m}+b_{n-1}\right) \tau_{n-1}^{m+1}=a_{m} \times\left|\overline{0}, \cdots, \overline{N-3}, \overline{N-2}{ }_{n-1}, N-2_{m+1}\right|,
$$

which yields eq. (59)

$$
\left(a_{m}+b_{n-1}\right) \tau_{n-1}^{m+1}=A(m) \times\left|0, \cdots, N-3, N-2_{n-1}, \widetilde{N-} 2_{m+1}\right| .
$$

We omit the proof of other difference formulas, since they can be proved in a similar manner.

\section{References}

[1] N. Matsuura: Book of Abstracts, DMHF2007: COE Conference on the Development of Dynamic Mathematics with High Functionality (Kyushu University, 2007) p.93.

[2] R. Hirota: J. Phys. Soc. Jpn. 43(1977) 1424.

[3] Y. Ohta, R. Hirota, S. Tsujimoto and T. Imai: J. Phys. Soc. Jpn. 62(1993) 1872.

[4] K. Kajiwara and J. Satsuma: J. Phys. Soc. Jpn. 60(1991) 506.

[5] K. Kajiwara, Y. Ohta and J. Satsuma: Phys. Lett. A180(1993) 249.

[6] A. Nagai, T. Tokihiro, J. Satsuma, R. Willox and K. Kajiwara: Phys. Lett. A234(1997) 301.

[7] R. Willox, T. Tokihiro and J. Satsuma: J. Math. Phys. 38(1997) 6455.

[8] R. Willox, T. Tokihiro and J. Satsuma: Chaos, Solitons and Fractals 11(2000) 121. 
[9] R. Hirota: J. Phys. Soc. Jpn. 50(1981) 3785.

[10] T. Miwa: Proc. Japan Acad. Ser. A Math. Sci. 58(1982) 9.

[11] M. Jimbo and T. Miwa: Publ. RIMS, Kyoto Univ. 19(1983) 943.

[12] I. Krichever, P. Wiegmann, A. Zabrodin: Commun. Math. Phys. 193(1998) 373.

[13] A. Mukaihira and S. Tsujimoto: J. Phys. A: Math. Gen. 37(2004) 4557.

[14] A. Mukaihira and S. Tsujimoto: J. Phys. A: Math. Gen. 39(2006) 779.

[15] K. Kajiwara and A. Mukaihira: J. Phys. A: Math. Gen. 38(2005) 6363.

[16] F. Nijhoff and H. Capel: Acta. Appl. Math. 39(1995) 133.

[17] D. Takahashi and R. Hirota: J. Phys. Soc. Jpn. 76(2007) 104007. 